\title{
EXTRATO DA CASCA DO FRUTO DO CACAUEIRO COMO FERTILIZANTE POTÁSSICO NO CRESCIMENTO DE MUDAS DE CACAUEIRO ${ }^{1}$
}

\author{
GEORGE ANDRADE SODRÉ ${ }^{2}$, MARCELA TONINI VENTURINI ${ }^{3}$, \\ DANIEL ORNELAS RIBEIRO ${ }^{4}$, PAULO CESAR LIMA MARROCOS
}

RESUMO - Em função da importância da casca do fruto como principal resíduo da cacauicultura e as possibilidades de seu uso, este trabalho teve como objetivos realizar análise de nutrientes do extrato orgânico obtido por lavagem do composto de casca e avaliar seu efeito como fertilizante potássico no solo e no crescimento de mudas de cacaueiro. O ensaio foi realizado em casa de vegetação, com aplicação de doses de extrato via solo, cultivo em tubetes e mudas seminais de cacaueiro. O delineamento experimental foi em blocos casualizados, com três repetições, e a unidade experimental formada por nove plantas crescidas individualmente em tubetes. Os tratamentos foram 5 doses de K: zero; 125; 250; 500 e 1.000 mg de $\mathrm{K} \mathrm{dm}^{-3}$ de solo. Após 120 dias da aplicação do extrato no solo, verificou-se que o pH, a saturação de bases e os teores disponíveis de $\mathrm{Ca}, \mathrm{Mg}, \mathrm{K}, \mathrm{Zn}$ e Mn aumentaram, e o teor de $\mathrm{Al}$ e Fe diminuíram. Os resultados também evidenciaram que a dose de $1.000 \mathrm{mg} \mathrm{K} \mathrm{dm}^{-3}$ de solo desequilibrou as relações $\mathrm{K} / \mathrm{Mg}$ e $\mathrm{K} / \mathrm{Ca}$ nas folhas com redução no crescimento das mudas. Mudas de cacaueiro apresentaram crescimento significativo em função de doses de K e, considerando a facilidade de produzir e aplicar o extrato da casca do fruto do cacaueiro, é possível usá-lo como fonte de K na produção de mudas de cacaueiro.

Termos para indexação: composto; mudas de cacau; potássio.

\section{EXTRACT FROM THE BARK OF COCOA FRUIT AS POTASSIUM FERTILIZER ON GROWTH OF COCOA SEEDLINGS}

\begin{abstract}
Considering the importance of the bark of cocoa as the main crop residue in cocoa farms, the objective of this study was to make analysis of nutrients from the organic extract obtained by washing the compost of the bark and to evaluate the effect of this extract as potassium fertilizer in soils and growth of cocoa seedlings. The experiment was conducted in a greenhouse with application of extract in the soil, growing in plastic tubes and seedlings of cocoa. The experimental design was in randomized blocks with three replications and experimental units consisting of nine plants grown individually in plastic tubes. The treatments were five $\mathrm{K}$ levels: zero, 125, 250, 500 and $1000 \mathrm{mg} \mathrm{K} \mathrm{dm}^{-3}$ of soil. After 120 days of the extract application in the soil, it was found that $\mathrm{pH}$, base saturation and $\mathrm{Ca}, \mathrm{Mg}, \mathrm{K}, \mathrm{Zn}$ and $\mathrm{Mn}$ available increased while the content of $\mathrm{Al}$ and Fe were reduced. The results also showed that the dose $1000 \mathrm{mg} \mathrm{K} \mathrm{dm}^{-3}$ of soil unbalanced the ratio $\mathrm{K} / \mathrm{Mg}$ and $\mathrm{K} / \mathrm{Ca}$ in leaves with reduced growth of seedlings. The application of bark extract increased significantly the available of $\mathrm{K}$ in the soil and concentration in plants. Cocoa seedlings showed growth in responses to different levels of $\mathrm{K}$ and, considering the easily of manufacture and applying the extract of the cacao bark, there is possible of the use as a source of $\mathrm{K}$ in the production of cocoa seedlings. Index terms: compost, cocoa seedlings, potassium.
\end{abstract}

\footnotetext{
1(Trabalho 168-11). Recebido em: 27-05-2011. Aceito para publicação: 25-07-2012.

${ }^{2}$ Pesquisador, Comissão Executiva do Plano da Lavoura Cacaueira \& Professor, Universidade Estadual de Santa Cruz - UESC, CP 07, CEP 45600-970, Itabuna-BA. E-mail: sodre@cepec.gov.br;

${ }^{3}$ Engenheira Agrônoma, Bolsista DTI-CNPq, Comissão Executiva do Plano da Lavoura Cacaueira, \& Mestranda, Programa de Pós-Graduação em Produção Vegetal da Universidade Estadual de Santa Cruz, Uesc - Ilhéus-BA. E-mail: cosalin2@yahoo.com.br

${ }^{4}$ Engenheiro Agrônomo, Bolsista FAPESPA, Comissão Executiva do Plano da Lavoura Cacaueira, CEP 45600-970, Itabuna-BA. E-mail:ornelasrib@hotmail.com;

${ }^{5}$ Pesquisador, Comissão Executiva do Plano da Lavoura Cacaueira \& Professor, Universidade Estadual de Santa Cruz - UESC, CP 07, CEP 45600-970, Itabuna-BA. E-mail: marrocos@cepec.gov.br
} 


\section{INTRODUÇÃO}

A região sul do Estado da Bahia configura-se como a maior fronteira nordestina de mata atlântica conservada, onde se cultivam mais de 500.000 ha com cacaueiros. Em função das dificuldades econômicas de se manejar uma cultura sobre o dossel da mata, como é o caso da cacauicultura, uma forma de reduzir custos e manter o interesse dos agricultores por essa atividade é o reaproveitamento de resíduos gerados nas áreas de produção.

Os resíduos agrícolas são classificados de acordo com os riscos ambientais e potencialidade de uso na agricultura. O exemplo mais conhecido de aproveitamento de resíduos na agricultura brasileira é o do bagaço da cana-de-açúcar, que atualmente é considerado um produto nobre no agronegócio do etanol e açúcar.

Na cultura do cacaueiro, o resíduo gerado em maior quantidade é a casca do fruto. Para produzir uma tonelada de amêndoas secas, são gerados aproximadamente 7 toneladas de casca fresca, com $80 \%$ de umidade. A casca é resultado da quebra de frutos para a extração das sementes, usadas para fabricação de chocolate, e quase sempre permanece no solo das plantações em forma de "casqueiros" que, se não tratados com fungicidas, constituem fonte de inóculo para fungos patogênicos às plantas do cacaueiro (MORORÓ, 2007).

Dentre as potencialidades de aproveitamento da casca do fruto,destacam-se o uso na alimentação animal (ABIOLA,1991), extração de pectinas (BERBERT, 1972), gomas, (FIGUEIRA et al., 1993) e produção de fertilizantes orgânicos por compostagem (CHEPOTE et al., 1990). Esse subproduto contém cerca de $4 \%$ de K em base seca (WOOD; LASS, 1985). Sodré et al. (2007) avaliaram as características químicas da fração mineral da casca do cacau e verificaram que o teor de potássio representava $73 \%$ dos elementos encontrados na fração cinza.

Durante a compostagem da casca do fruto, verifica-se a liberação de extrato líquido, escuro e inodoro. Sodré et al. (2007) realizaram análise química desse extrato e encontraram elevada concentração de $\mathrm{K}$ e condutividade elétrica (CE) de 1,9 dS m${ }^{-1}$, após diluição de $1 \mathrm{~g}$ do extrato seco a $60{ }^{\circ} \mathrm{C}$ em 100 $\mathrm{mL}$ de água destilada.

A produção de mudas de cacaueiro é uma importante etapa para a implantação de novas áreas, adensamento de stands ou substituição de plantas que apresentam baixa produção. De acordo com Sodré e Marrocos (2009), quando as mudas de cacaueiro são produzidas com baixa tecnologia, os plantios não conseguem alcançar o potencial produtivo máximo.
Assim, para produzir mudas com qualidade elevada, é necessário selecionar porta-enxertos que aumentem a eficiência de absorção e transporte de nutrientes. Nesse contexto, Sodré e Marrocos (2009) recomendam uso de sementes de cacaueiro da variedade 'Comum' oriundas de polinização aberta.

No que se refere à nutrição mineral do cacaueiro, sabe-se que a demanda de $\mathrm{K}$ é alta na fase de produção; no entanto, existem poucos estudos na fase de produção das mudas. Num experimento em casa de vegetação e usando resíduo de casca de coco como fonte de K, Baon (2009) obteve maior crescimento de mudas de cacaueiros na dose de 700 mg de K para 2,5 kg de solo. Por outro lado, Gama-Rodrigues et al. (1995) avaliaram fontes minerais de $\mathrm{K}$ em mudas seminais e verificaram que o $\mathrm{KCl}$ foi significativamente superior em relação ao $\mathrm{K}_{2} \mathrm{SO}_{4}$ e Kalsilita para os parâmetros taxa respiratória e fotossíntese líquida.

O trabalho teve como objetivos caracterizar o extrato orgânico obtido por lavagem do composto de casca do fruto e avaliar o efeito do uso do extrato como fertilizante potássico no solo e no crescimento de mudas de cacaueiro.

\section{MATERIAL E MÉTODOS}

O trabalho foi realizado em casa de vegetação coberta com telhas de fibra de vidro, pé- direito medindo $5 \mathrm{~m}$ e laterais de sombrite de cor preta, proporcionando $50 \%$ de sombreamento, localizada na Comissão Executiva do Plano da Lavoura Cacaueira - Ceplac, Ilhéus-BA. O experimento foi realizado entre os meses de abril e agosto de 2010. Inicialmente, foi preparado o composto de casca do fruto e obtido o extrato, em seguida foi realizado ensaio para verificar o efeito do uso do extrato como fonte de potássio.

Na preparação do composto, a casca do fruto do cacaueiro foi inicialmente picada em pedaços retangulares entre 1,5 e 2,5 cm de largura. O composto foi preparado em forma de pilha, com as dimensões de 2,5 m de comprimento e 1,5 m de largura, e 1,0 m de altura, sob lona de polietileno. Foram realizados revolvimentos a cada oito dias até quando não foi mais verificado aquecimento da massa, e a temperatura situou-se entre 28 e $30^{\circ} \mathrm{C}$, o que ocorreu aos 90 dias, quando então o material foi considerado estabilizado.

Para a obtenção do extrato, trinta litros de composto, com aproximadamente $70 \%$ de umidade, foram espalhados sobre uma peneira de $40 \mathrm{~cm}$ de largura, $1 \mathrm{~m}$ de comprimento e $4 \mathrm{~mm}$ de malha e, em seguida, foram adicionados lentamente 3 litros 
de água destilada. Sob a peneira, foi colocada uma calha para a coleta do extrato líquido. Após a coleta, o líquido foi seco em estufa a $60^{\circ} \mathrm{C}$, por 48 horas, e em seguida moído e passado em peneira de $1,0 \mathrm{~mm}$. Em amostras do extrato seco, e segundo metodologia descrita por Embrapa (1999), foram determinados os teores de C, N, P, K, Ca, Mg, Cu, Fe, Mn e Zn, com três repetições.

Para avaliar o uso do extrato como fertilizante potássico,utilizou-se solo da camada de $0-20 \mathrm{~cm}$, coletado em um Argissolo Vermelho-Amarelo, distrófico, que foi inicialmente seco ao ar, peneirado em malha de $2 \mathrm{~mm}$ e analisado quimicamente, conforme metodologia descrita por Embrapa (1999), apresentando $\mathrm{pH} \mathrm{H}_{2} \mathrm{O}=5,2, \mathrm{Al}\left(\mathrm{KCl} 1 \mathrm{~mol} \mathrm{~L}^{-1}\right)=0,5 \mathrm{cmol}_{c}$ $\mathrm{dm}^{-3}$, P Mehlich $-1=2,0 \mathrm{mg} \mathrm{dm}^{-3}$, K Mehlich $-1 \stackrel{\mathrm{c}}{=}$ $0,09 \mathrm{cmol}_{\mathrm{c}} \mathrm{dm}^{-3}$, Ca $\left(\mathrm{KCl} 1 \mathrm{~mol} \mathrm{~L}^{-1}\right)=2,1 \mathrm{cmol}_{\mathrm{c}} \mathrm{dm}^{-3}$, $\mathrm{Mg}\left(\mathrm{KCl}_{1}^{\mathrm{c}} \mathrm{mol} \mathrm{L}^{-1}\right)=0,9 \mathrm{cmol}_{\mathrm{c}} \mathrm{dm}^{-3}, \mathrm{CTC}_{\mathrm{pH} 7}=9,7$ $\mathrm{cmol}_{\mathrm{c}} \mathrm{dm}^{-3} \mathrm{Fe}=101 \mathrm{mg} \mathrm{dm}^{-3}, \mathrm{Zn}=2,3 \mathrm{mg} \mathrm{dm}^{-3}, \mathrm{Cu}$ $=1,0 \mathrm{mg} \mathrm{dm}^{-3}$ e Mn $=8,3 \mathrm{mg} \mathrm{dm}^{-3}$.

O delineamento experimental utilizado foi em blocos casualizados, com três repetições, e a unidade experimental foi formada por nove plantas crescidas individualmente em tubetes. Os tratamentos foram constituídos por 5 doses: zero; 125; 250; 500 e 1.000 $\mathrm{mg}$ de $\mathrm{K} \mathrm{dm}^{-3}$ de solo na forma de extrato da casca do fruto. A quantidade de extrato usada para atender às doses foi calculada a partir do resultado da análise química para K (Tabela 1).

Após a aplicação, o extrato foi homogeneizado ao solo. Utilizaram-se sacos plásticos individuais para cada dose. Antes do plantio, o solo recebeu adubação de base, adicionando-se para cada $\mathrm{dm}^{3}$ $250 \mathrm{mg}$ de N, $150 \mathrm{mg}$ de P, 5,0 mg de Zn, 5,0 mg de $\mathrm{Cu}, 1,0 \mathrm{mg}$ de B, 1,0 mg de Mn e 0,1 mg de Mo. Após a adubação, o solo foi umedecido para $70 \%$ da capacidade de campo e incubado por 2 semanas.

Sementes do porta-enxerto "comum" foram inicialmente germinadas em mistura de areia e serragem, na proporção volumétrica 1:1 (v:v) e, quando foi verificada a emissão da radícula, foram transplantadas à profundidade de $2 \mathrm{~cm}$ em tubetes de $288 \mathrm{~cm}^{3}$, previamente preenchidos com o solo incubado. Os tubetes apresentavam $18 \mathrm{~cm}$ de comprimento, $5 \mathrm{~cm}$ de abertura superior, continham orifício de drenagem de $0,5 \mathrm{~cm}$ na base e foram dispostos em suportes de isopor suspensos a $5 \mathrm{~cm}$, em bancada de madeira.

Durante o crescimento, as plantas foram mantidas em casa de vegetação e irrigadas 3 vezes por semana com água destilada para manter o solo em aproximadamente 70\% da capacidade de campo.

Aos 30; 60 e 90 dias após o plantio, cada tubete foi fertilizado com $10 \mathrm{~mL}$ de solução recomendada para mudas de cacaueiros por Sodré et al. (2010). Essa solução continha: $5 \%$ de $\mathrm{N} ; 12 \%$ de $\mathrm{P}_{2} \mathrm{O}_{5} ; 12 \%$ de Ca;1,5 \% de Mg;5\% de S, e os micronutrientes Zn 2\%; B 1,5\%;Cu 0,5\%, Mn 0,5\%; Mo 0,2\% e Fe 0,1\%.

Após 120 dias de semeadura, efetuou-se a medição do diâmetro do caule (DC) a $5 \mathrm{~cm}$ do coleto e a altura das plantas (AP) do coleto até a ponta do último lançamento. Em seguida, as plantas foram cortadas na altura do coleto, e as folhas, separadas para a medição da área foliar (AF), utilizando-se do medidor eletrônico modelo LI- 3100. Folhas, raízes e caule foram lavados e secos em estufa a $65^{\circ} \mathrm{C}$ até atingir peso constante, e avaliadas as variáveis biológicas: massa seca da parte aérea (MSPA), massa seca da raiz pivotante (MSRP) e secundária (MSRS). No material seco das folhas, foram analisados os teores de N, P, K, Ca e Mg, segundo metodologia descrita pela Embrapa (1999).

Os resultados foram submetidos à análise de variância e de regressão, e foram ajustadas em função de doses de K. Foram aceitos os modelos que apresentaram coeficientes significativos a até $5 \%$, pelo teste $\mathrm{F}$, e o maior coeficiente de determinação $\left(\mathrm{R}^{2}\right)$.

\section{RESULTADOS E DISCUSSÃO}

A aplicação de doses de $\mathrm{K}$ via extrato da casca do fruto do cacaueiro no solo elevou o $\mathrm{pH}$, a saturação de bases e os teores disponíveis de $\mathrm{Ca}$, Mg, K, Zn e Mn. Os teores disponíveis de $\mathrm{Cu}$ e Zn praticamente não se alteraram, enquanto $\mathrm{Al}$ e $\mathrm{Fe}$ foram reduzidos (Tabela 2).

A redução no teor de Fe, verificado principalmente para a dose de $1.000 \mathrm{mg}$, pode ser atribuída à complexação do Fe por substâncias húmicas, provavelmente, contidas no extrato. No que se refere à disponibilidade de nutrientes em solos tratados com matéria orgânica, pesquisas demonstraram que ligantes orgânicos hidrossolúveis com grupos funcionais hidroxílicos e carboxílicos (MIYAZAWA et al., 1992), e compostos orgânicos hidrossolúveis de baixo peso molecular (FRANCHINI et al., 1999), liberados por resíduos vegetais e presentes em extratos vegetais, são os responsáveis por alterações químicas nos solos.

Considerando que o solo não recebeu calagem, a redução no teor de $\mathrm{Al}$ pode ser atribuída tanto ao aumento do $\mathrm{pH}$, quanto à complexação do Al por componentes orgânicos presentes no extrato. Redução da disponibilidade de Al e complexação por compostos orgânicos foram verificadas na cultura do trigo (MIYAZAWA et al.,1992). Deve-se também considerar a possibilidade de perda da estabilidade de agregados e consequente lixiviação do $\mathrm{Al}$ e outros elementos via orifício de drenagem do tubete. Nesse 
contexto, Benites e Mendonça (1998) observaram que a adição de substâncias húmicas pode alterar o estado de agregação do solo e está relacionada com o aumento da condutividade elétrica e a presença de sais.

A saturação por Al foi reduzida entre as doses de zero e $1.000 \mathrm{mg}$ de $\mathrm{K}$ de 24 para 5\%. Considerando que o nível crítico para Al tolerado por mudas de cacaueiro é de 15\% (BALIGAR; FAGERIA, 2005), os resultados indicam que o uso do extrato como fonte de $\mathrm{K}$, até a dose de $500 \mathrm{mg}$, contribuiu na melhoria dessa condição química e também possibilitou maior crescimento das mudas.

Foi verificado ajuste linear significativo para o K disponível no solo em função das doses (Tabela 2), indicando que o K contido no extrato foi disponibilizado para o solo. Isso pode ser explicado porque o K não faz parte de moléculas, e estando na forma livre nos tecidos vegetais (KLEPER; ANGHINONI, 1995) é facilmente liberado,tornando-se disponível às plantas.

A relação $\mathrm{K} / \mathrm{Mg}$ no solo variou entre 0,18 e 1,1 para as doses de 0 e $1.000 \mathrm{mg}$, respectivamente. No que se refere à relação $\mathrm{K} / \mathrm{Mg}$ no solo, Boyer (1972) sugere para cultivos tropicais e subtropicais valores entre 0,25 e 0,33 . Considerando o forte antagonismo que ocorre entre $\mathrm{K} \mathrm{e} \mathrm{Mg}$ no processo de absorção pela planta, Py et al. (1987) afirmaram que a relação $\mathrm{K} / \mathrm{Mg}$ no solo não deve ser maior do que 1,0. Assim, verifica-se pelos resultados deste trabalho que, para manter a relação $\mathrm{K} / \mathrm{Mg}$ dentro da faixa recomendada, a dose de $\mathrm{K}$ a ser aplicada em mudas de cacaueiro deverá variar entre 125 e 500 $\mathrm{mg} \mathrm{dm} \mathrm{dm}^{-3}$ de solo.

O extrato aplicado no solo como fonte de $\mathrm{K}$ resultou em aumento significativo no teor de $\mathrm{K}$ e nível crítico foliar para atingir $90 \%$ da produção de massa seca da parte aérea de $32,1 \mathrm{~g} \mathrm{~kg}^{-1}$. O coeficiente de determinação alto $\left(\mathrm{R}^{2}=0,96\right)$ do modelo quadrático apresentado na Tabela 3 indica alta preditividade do modelo ajustado. Respostas quadráticas à aplicação de potássio em mudas de maracujazeiro foram encontradas por Prado et al. (2004),que verificaram nível crítico foliar para o K em mudas de 38,5 $\mathrm{g} \mathrm{kg}^{-1}$.

Não foram encontradas significâncias pelo teste F para teores foliares de Ca e Mg em função das doses de K. Para Ca e Mg, a dose de 1.000 mg reduziu o teor desses elementos em comparação com a dose zero em 27 e 14\%, respectivamente (Tabela 3). A redução nos teores totais de Ca e Mg nas folhas, especialmente na dose de $1.000 \mathrm{mg}$, pode ser atribuída ao antagonismo entre esses nutrientes, mas, especificamente, à maior concentração do íon $\mathrm{K}$ na solução do solo e sua relativa facilidade em atravessar a plasmalema, via canais iônicos (FERNANDES; SOUZA, 2006).

Os resultados encontrados no trabalho sugerem que houve consumo de luxo para o K e consequente desequilíbrio com o Ca e o Mg. Redução dos teores foliares de Ca e Mg, em resposta ao consumo de luxo de $\mathrm{K}$, é comum em diferentes cultivos, a exemplo de $\mathrm{Mg}$ em soja (MASCARENHAS et al., 1980) e Ca em feijão (NARWAL et al., 1985). Nesse contexto, Souza et al. (2002) verificaram reduções nas concentrações de cálcio e magnésio na folha ' $\mathrm{D}$ ' do abacaxizeiro 'Pérola', em decorrência da adubação com doses crescentes de potássio, atribuindo esse fato às competições que ocorrem no processo de absorção desses nutrientes. $\mathrm{O}$ antagonismo $\mathrm{Ca} / \mathrm{K}$ e $\mathrm{Mg} / \mathrm{K}$, em geral, é mais acentuado com o aumento da idade da folha, sugerindo que as folhas velhas são usadas como depósitos do excesso e como fontes para a remobilização do $\mathrm{K}$.

As variáveis massa seca da parte aérea (MSPA), altura da planta (AP) e diâmetro do caule (DC) mostraram respostas quadráticas e significativas às doses de K (Tabela 4). Para MSPA, o nível crítico foi de 1,81 g/tubete para a dose de 391,6 mg de K $\mathrm{dm}^{-3}$ de solo (Figura 1).

Considerando que porta-enxertos de cacaueiros necessitam atingir $10 \mathrm{~mm}$ de diâmetro do caule para que seja efetuada a enxertia, aumentos significativos do diâmetro são importantes e, nesse contexto, a fertilização com extrato e, consequentemente, com o K nele contido pode contribuir para a redução do tempo necessário até o momento da enxertia e, com isso, reduzir custos na produção das mudas de cacaueiro. 
TABELA 1- Análise química do extrato da casca do fruto do cacaueiro. Ceplac-BA, Ilhéus, 2010.

\begin{tabular}{|c|c|c|c|c|c|c|c|c|c|}
\hline $\mathrm{C}^{*}$ & $\mathrm{~N}$ & $\mathrm{P}$ & $\mathrm{K}$ & $\mathrm{Ca}$ & $\mathrm{Mg}$ & $\mathrm{Fe}$ & $\mathrm{Zn}$ & $\mathrm{Cu}$ & $\mathrm{Mn}$ \\
\hline & & $-g$ & & & 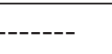 & & ני-י-יני & $8-$ & - \\
\hline $\begin{array}{l}400,0 \\
( \pm 4,4)\end{array}$ & $\begin{array}{c}27,4 \\
( \pm 6,5)\end{array}$ & $\begin{array}{c}2,6 \\
( \pm 0,5)\end{array}$ & $\begin{array}{c}145,9 \\
( \pm 58,2)\end{array}$ & $\begin{array}{c}13,1 \\
( \pm 4,5)\end{array}$ & $\begin{array}{c}14,0 \\
( \pm 5,0)\end{array}$ & $\begin{array}{c}888 \\
( \pm 406,8)\end{array}$ & $\begin{array}{c}1690 \\
( \pm 825,2)\end{array}$ & $\begin{array}{c}74 \\
( \pm 30,5)\end{array}$ & $\begin{array}{c}234 \\
( \pm 98,6)\end{array}$ \\
\hline
\end{tabular}

* Dados médios (desvio- padrão, $\mathrm{n}=3$ ).

TABELA 2- Características do solo 120 dias após a aplicação de doses de K via extrato da casca do fruto do cacaueiro e plantio de mudas de cacaueiro. Ceplac-BA, Ilhéus, 2010.



**Significativo a $1 \%$ de probabilidade; ${ }^{(1)} \hat{\mathrm{y}}=0,0009 \mathrm{x}+0,006\left(\mathrm{R}^{2}=0,97 * *\right) . \mathrm{SB}=$ soma de bases; $\mathrm{T}=$ capacidade de troca de cátions a pH 7; V = saturação por bases; $m$ = saturação por alumínio.

TABELA 3- Teores de N, P, K, Ca, Mg e relações K/Ca, K/Mg em folhas de mudas de cacaueiro em função de doses de K aplicadas via extrato da casca do fruto do cacaueiro. Ceplac-BA, Ilhéus, 2010.

\begin{tabular}{|c|c|c|c|c|c|c|c|}
\hline Doses de K & $\mathrm{N}$ & $\mathrm{P}$ & $\mathrm{K}^{*}$ & Ca & $\mathrm{Mg}$ & K/Ca & $\mathrm{K} / \mathrm{Mg}$ \\
\hline $\mathrm{mg} \mathrm{dm}^{-3}$ & & $\ldots$ & .......g & 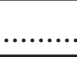 & $\ldots$ & & \\
\hline 0 & $18,9^{\text {ns }}$ & $2,1^{\mathrm{ns}}$ & $21,7^{1}$ & $5,1^{\text {ns }}$ & $2,5^{\text {ns }}$ & 4,3 & 8,7 \\
\hline 125 & 17,7 & 2,1 & 24,7 & 4,3 & 2,6 & 5,7 & 9,5 \\
\hline 250 & 17,1 & 1,9 & 30,3 & 4,3 & 2,5 & 7,0 & 12,1 \\
\hline 500 & 17,3 & 2,1 & 32,4 & 4,4 & 2,3 & 7,4 & 14,1 \\
\hline 1000 & 18,4 & 1,8 & 31,6 & 3,7 & 2,0 & 8,5 & 15,8 \\
\hline CV\% & 5 & 7 & 6 & 8 & 6 & - & - \\
\hline
\end{tabular}

*Significativo a 5\% de probabilidade. ns = não significativo. ${ }^{(1)} \hat{y}=-0,8014 x^{2}+7,5626 x+14,279\left(R^{2}=0,96 *\right)$.

TABELA 4 - Características das mudas de cacaueiro em função de doses de K aplicadas via extrato da casca do fruto do cacaueiro em tubetes de $288 \mathrm{~cm}^{3}$. Ceplac-BA, Ilhéus, 2010.

\begin{tabular}{|c|c|c|c|c|c|c|}
\hline Doses de $\mathrm{K}$ & MSRP & MSRS & MSPA $^{*}$ & $\mathrm{AF}$ & $\mathrm{AP}^{*}$ & $\mathrm{DC}^{*}$ \\
\hline $\mathrm{mg} \mathrm{dm}^{-3}$ & ---------- & ---g --- & ---------- & $\mathrm{cm}^{2}$ & $\mathrm{Cm}$ & $\mathrm{Mm}$ \\
\hline 0 & $0,33^{\text {ns }}$ & $0,15^{\mathrm{ns}}$ & $1,51^{1}$ & $336,1^{\mathrm{ns}}$ & $22,3^{2}$ & $3,9^{3}$ \\
\hline 125 & 0,41 & 0,17 & 1,83 & 339,3 & 25,8 & 4,1 \\
\hline 250 & 0,36 & 0,22 & 1,88 & 347,9 & 24,7 & 4,2 \\
\hline 500 & 0,38 & 0,21 & 1,78 & 306,3 & 24,3 & 4,0 \\
\hline 1000 & 0,35 & 0,16 & 1,57 & 303,8 & 23,3 & 3,9 \\
\hline CV \% & 17 & 11 & 14 & 16 & 12 & 6 \\
\hline
\end{tabular}

*Significativo a $5 \%$ de probabilidade; ns = não significativo. ${ }^{(1)} \hat{y}=-0,00000119 x^{2}+0,00114029 x+1,60276923\left(R^{2}=0,69 *\right) ;{ }^{(2)} \hat{y}=$ $-0,6032 x^{2}+3,6635 x+19,778\left(R^{2}=0,68^{*}\right) ;{ }^{(3)} \hat{y}=-0,0654 x^{2}+0,3827 x+3,654\left(R^{2}=0,83^{*}\right)$. MSRP = Massa seca da raiz pivotante; MSRS = Massa seca da raiz secundária; MSPA = Massa seca da parte aérea; AF = Área foliar; AP = Altura da planta; DC = Diâmetro do caule. 


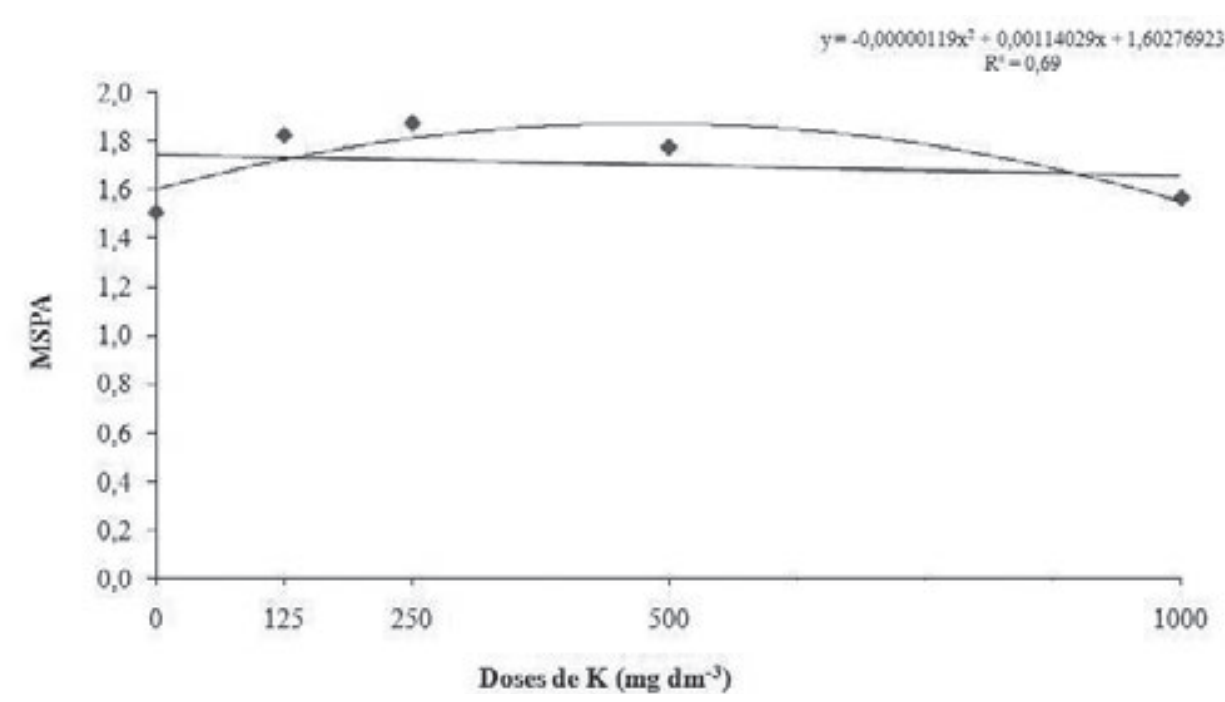

FIGURA 1 - Massa seca da parte aérea de mudas de cacaueiro em função de doses de K aplicadas via extrato da casca do fruto do cacaueiro.()

\section{CONCLUSÕES}

1-A massa seca da parte aérea de mudas de cacaueiro é aumentada significativamente em função de doses de potássio aplicadas no solo via extrato da casca do fruto com nível foliar crítico de 32,1 $\mathrm{g} \mathrm{kg}^{-1}$.

2-É possível usar o extrato da casca do fruto do cacaueiro como fonte de potássio na produção de mudas de cacaueiro na dose de 391,6 mg de K $\mathrm{dm}^{-3}$ de solo.

\section{AGRADECIMENTOS}

Ao Conselho Nacional de Desenvolvimento Científico e Tecnológico (CNPq), pelo financiamento do projeto de pesquisa No $574898 / 2008-5$ e bolsa de DTI.

\section{REFERÊNCIAS}

ABIOLA, S. S.; TEWE, O. O. Chemical evaluation of cocoa by-products. Tropical Agriculture, Trinidad, v. 68, n. 4, p. 335-336, 1991.

BALIGAR, V. C.; FAGERIA, N. K. Aluminum influence on growth and uptake of micronutrients by cacao. Journal of Food, Agriculture \& Environment, Helsinki, v. 3, n. 3-4, p. 173-177, 2005.
BAON, J. B. Use of Plant Derived Ash as Potassium Fertilizer and Its Effects on Soil Nutrient Status and Cocoa Growth. Jurnal Tanah Tropika, Bandar Lampung, v. 14, n. 3, p. 185-193, 2009.

BENITES, V. M.; MENDONÇA, E. S. Propriedades eletroquímicas de um solo eletropositivo influenciadas pela adição de diferentes fontes de matéria orgánica. Revista Brasileira de Ciência do Solo, Viçosa, MG, v. 22, n. 2, p. 215-221, 1998.

BERBERT, P. R. Estudo da pectina do mel e da casca do fruto do cacau. Revista Theobroma, Ilhéus, v. 2, n. 49, p. 51, 1972.

BOYER, J. Soil potassium. In: COMMITTEE ON TROPICAL SOILS. Soils of the humid tropics. Washington: National Academy Science, 1972. p. 102-135.

CHEPOTE, R. E.; SANTANA, J. L. C.; De LEON, F. Como utilizar composto de casca de cacau na adubação do cacaueiro. Difusão Agropecuária, Ilhéus, v. 2, n. 1, p. 11-17, 1990.

EMBRAPA - Empresa Brasileira de Pesquisa Agropecuária. Manual de análises químicas de solos, plantas e fertilizantes. Brasília: Embrapa Informação Tecnológica, 1999. 370p. 
FERNANDES, M. S.; SOUZA, M. R. Absorção de Nutrientes. In: FERNANDES, M. S. (Ed.). Nutrição mineral de plantas. Viçosa: Sociedade Brasileira de Ciencia do Solo, 2006. p. 139.

FIGUEIRA, A.; JANICK, J.; BEMILLER, J. N. New products from Theobroma cacao: Seed pulp and pod gum. In: JANICK, J. and SIMON, J. E. (Ed.). New crops. New York: Wiley, 1993. p. 475-478.

FRANCHINI, J. C.; MALAVOLTA, E.; MIYAZAWA, M.; PAVAN, M. A. Alterações químicas em solos ácidos após a aplicação de resíduos vegetais. Revista Brasileira de Ciência do Solo, Viçosa-MG, v. 23, n. 3, p. 533-542, 1999.

GAMA-RODRIGUES, A. C.; VALLE, R. R.; ROSSIELLO, R. O. P. Crescimento, trocas gasosas e relações hídricas de plântulas de cacau em função de diferentes fontes de potássio. Revista Brasileira de Ciência do Solo, Viçosa-MG,v. 19, n. 3, p. 387393, 1995.

KLEPKER, D.; ANGHINONI, I. Características físicas e químicas do solo afetadas por métodos de preparo e modos de adubação. Revista Brasileira de Ciência do Solo, Viçosa-MG, v.19, n. 3, p. 395401, 1995.

MASCARENHAS, H. A. A.; NEPTUNE, A. M. L.; MURAOKA, T.; BULISANI, E. A.; HIROCE, R. Absorção de nutrientes por cultivares de soja (Glicine max (L.) Merril). Revista Brasileira de Ciência do Solo, Viçosa-MG, v. 4, p. 92-96, 1980.

MIYAZAWA, M.; CHIERICE, G. O.; PAVAN, M. A. Amenização da toxicidade de alumínio às raízes do trigo pela complexação com ácidos orgânicos. Revista Brasileira de Ciência do Solo, Viçosa-MG, v. 16, n. 2, p. 209-215, 1992.

MORORÓ, R. C. Aproveitamento de derivados, subprodutos e resíduos do cacau. In: VALLE, R. R. (Ed.). Ciência, tecnologia e manejo do cacaueiro. Ilhéus: Ceplac, 2007. p. 371- 421.
NARWAL, R. P.; KUMAR, V.; SINGH, J. P. Potassium and magnesium relationship in cowpea (Vigna unguiculata (L.) Walp.). Plant and Soil, Netherlands, v. 86, n. 1, p. 129 - 134, 1985.

PRADO, R de M.; BRAGHIROLLI, L. F.; NATALE, W.; CORRÊA, M. C. de M.; ALMEIDA, E. V. Aplicação de potássio no estado nutricional e na produção de matéria seca de mudas de maracujazeiro-amarelo. Revista Brasileira de Fruticultura, Jaboticabal, v. 26, n. 2, p. 295 - 299, 2004.

PY, C., LACOEUILHE. J. J.; TEISON, C. The pineapple, cultivation and uses. Paris: GP Maisonneuve et Larose, 1987.

SODRÉ, G. A.; MARROCOS, P. C. L.; PACHECO, R. G.; FONTES, M. I. L.; SERÓDIO, M. H.; LEMOS, M. T. O. Atributos químicos do extrato da casca do cacaueiro. In: CONGRESSO BRASILEIRO DE CIÊNCIA DO SOLO, 31., 2007, Gramado. Anais... Viçosa: Sociedade Brasileira de Ciência do Solo, 2007. CD-ROM.

SODRÉ, G. A.; MARROCOS, P. C. L. Manual da produção vegetativa de mudas de cacaueiro. Ilhéus: Editus, 2009. 46p.

SODRÉ, G. A.; MARROCOS, P. C. L.; PACHECO, R. G. Qualidade de mudas de cacaueiro produzidas por estaquia e transportadas sem substrato. Magistra, Cruz das Almas, v. 22, n. 2, p. 119 - 122, 2010.

SOUZA, L. F. da S.; GONÇALVES, N. B.; R. C.; CALDAS, A. G. S.; MEDINA, V. M. Influência da adubação potássica nos teores foliares de nutrientes do abacaxizeiro 'Pérola'. In: CONGRESSO BRASILEIRO DE FRUTICULTURA, 17., 2002, Belém. Anais... Jaboticabal: Sociedade Brasileira de Fruticultura, 2002. CD-ROM.

WOOD, G. A. R.; LASS, R. A. Cocoa. $4^{\text {th }}$ ed. England: Longman, Essex, 1985. 620p. 